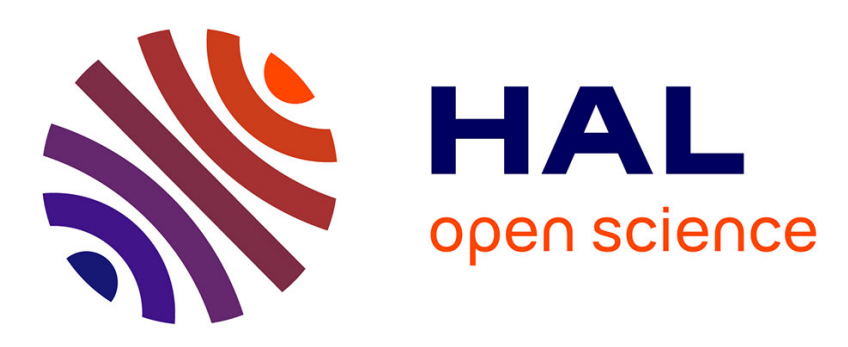

\title{
La Recherche en Chimie aux Frontières de la Connaissance
}

Dominique Massiot, Claire-Marie Pradier, Jacques Maddaluno, Jean-François Tassin, Joël Moreau, Mehran Mostafavi, Anne Imberty, Pascal Breuilles, Catherine Larroche

\section{To cite this version:}

Dominique Massiot, Claire-Marie Pradier, Jacques Maddaluno, Jean-François Tassin, Joël Moreau, et al.. La Recherche en Chimie aux Frontières de la Connaissance. L'industrie Chimique: quel avenir en France?, 2015, Annales des Mines, 2015, Annales des Mines. Réalités Industrielles. hal-01228736

\section{HAL Id: hal-01228736 https://hal.science/hal-01228736}

Submitted on 13 Nov 2015

HAL is a multi-disciplinary open access archive for the deposit and dissemination of scientific research documents, whether they are published or not. The documents may come from teaching and research institutions in France or abroad, or from public or private research centers.
L'archive ouverte pluridisciplinaire HAL, est destinée au dépôt et à la diffusion de documents scientifiques de niveau recherche, publiés ou non, émanant des établissements d'enseignement et de recherche français ou étrangers, des laboratoires publics ou privés. 


\section{La Recherche en Chimie aux Frontières de la Connaissance}

Dominique Massiot, Claire-Marie Pradier, Jacques Maddaluno, Jean-François Tassin, Joël Moreau, Mehran Mostafavi, Anne Imberty, Pascal Breuilles et Catherine Larroche

$$
\text { INC - Institut de Chimie du CNRS }
$$

L'Unité Mixte de Recherche est la brique élémentaire, souple et pérenne d'un paysage de la recherche en évolution rapide. De nombreux dispositifs viennent structurer l'activité de ces équipes pour faire émerger des pôles de recherche, consolider le maillage des réseaux nationaux, identifier les points forts, mutualiser des équipements de haut niveau et promouvoir les collaborations académiques et industrielles. La diversité de l'activité des chimistes se place dans un continuum thématique dépassant les frontières disciplinaires et traversant les mondes académiques et industriels. La dichotomie entre les structures disciplinaires de nos tutelles et l'affichage de défis socio-économiques par les agences de financement doivent permettre aux acteurs de mettre en œuvre des recherches de très haut niveau tout en favorisant l'émergence et la description de nouveaux concepts en rupture qui permettront de construire la chimie de demain.

La chimie, cette science qui étudie la matière et ses transformations, entreprend d'imaginer et de créer de nouveaux objets, de décrire et de prévoir la variété et la complexité des assemblages atomiques ou moléculaires, d'en comprendre, d'en ajuster et d'en optimiser les propriétés. La recherche aux frontières de la connaissance s'attache tout particulièrement à faire émerger de nouveaux concepts en rupture avec les idées établies. Les champs d'étude de la chimie couvrent les différents états de la matière (gazeux, liquide et solide), leurs structures comme leurs dynamiques et leurs interfaces. En synergie avec la physique, la biologie, la pharmacie, l'environnement et l'écologie, grâce à la modélisation et à la simulation, aux spectroscopies, aux méthodes analytiques et à leurs applications, la chimie s'est toujours enrichie d'interactions fortes avec les disciplines voisines. Ses objets d'étude couvrent donc tout un panorama allant des recherches de base aux applications finalisées ou aux procédés industriels, dans un continuum thématique qui ignore les frontières disciplinaires.

Le panorama de l'écosystème de la recherche française a connu une évolution rapide avec des modifications marquantes dans les structures, les outils, les indicateurs, les objectifs et le mode de financement des laboratoires. Ces évolutions sont bien sûr impulsées par l'État (lois, budget, agences de financement ou agences d'évaluation, Stratégie Nationale de la Recherche), l'Europe (PCRD, H2020), les organismes et établissements, les industriels (les grandes entreprises comme les PME) et elles sont mises en œuvre par les acteurs euxmêmes: chercheurs (universités et organismes), ingénieurs, techniciens et directions des diverses unités.

La mise en place de l'autonomie des universités a engendré d'importantes modifications au contexte général de la recherche académique. Ainsi, le Plan d'Investissement d'Avenir (PIA1) (2010-2011), dans le cadre d'un processus compétitif de sélection, a mis en place les 
Initiatives d'eXcellence (IdeX) et un ensemble de structures projets (LabeX, EquipeX, ITE, IRT...).

Ce processus débouche aujourd'hui sur une structuration territoriale en COMmunautés d'Universités et d'Établissements (COMUE), qui s'accompagne de reconfigurations structurelles, dans le cadre de politiques de site impliquant tous les partenaires, locaux comme nationaux. Cela conduira sans doute à l'identification d'un nombre limité de sites majeurs ou de réseaux de recherche et d'enseignement supérieur interdisciplinaires ayant des spécificités clairement identifiées tant sur le plan de la recherche académique que sur celui de la valorisation et de son impact socio-économique. Les dernières étapes de ce processus sont les appels PIA en cours ou annoncés.

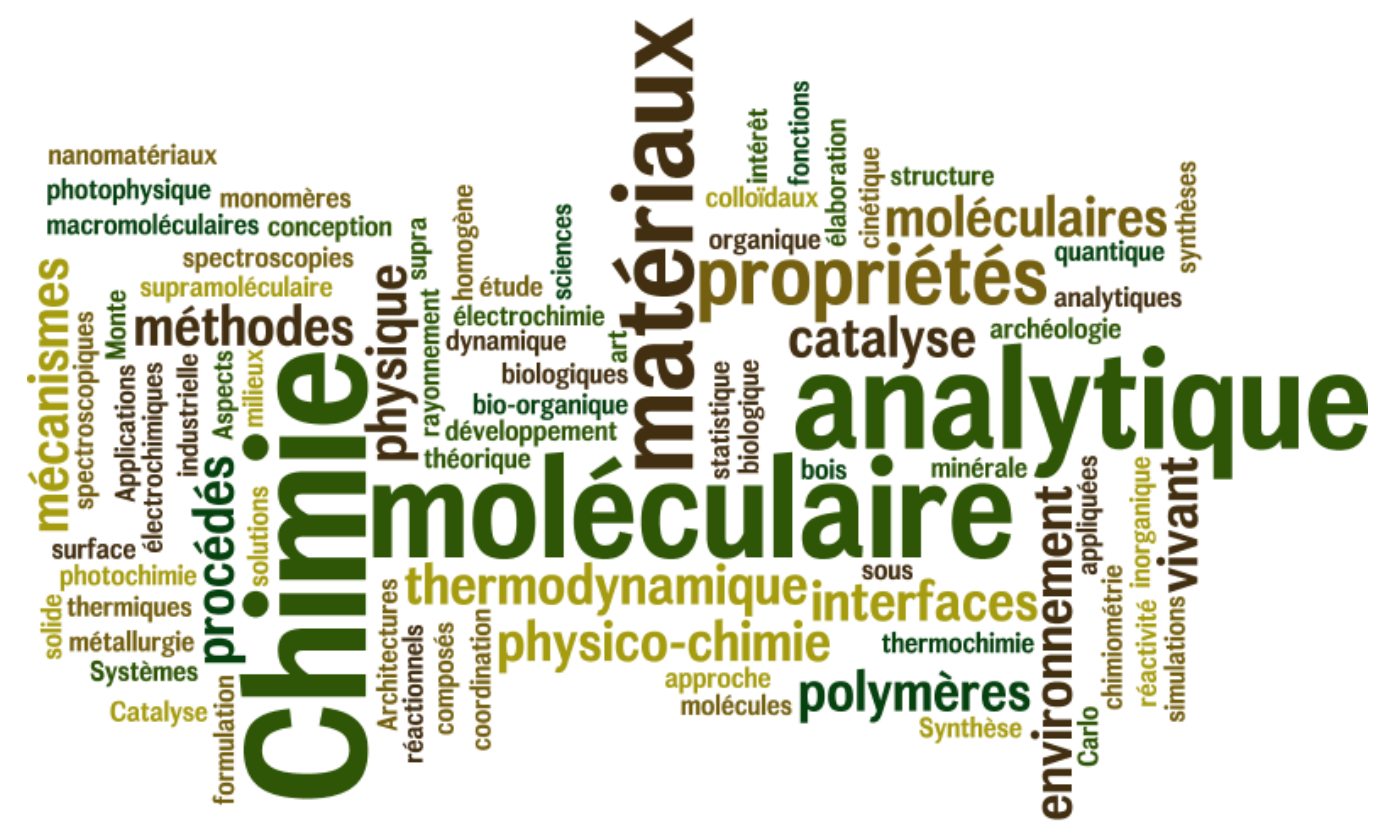

Figure 1 : Mots clés de la chimie académique à partir des intitulés des sections 31 à $33 d u$ Conseil National des Universités (CNU) et des Sections 11 à 16 du Comité National de la Recherche Scientifique (CoNRS)

Parallèlement, l'Agence Nationale de la Recherche (ANR), qui est une des sources importantes de financement de la recherche de base malgré un budget et des taux de succès trop limités, a opéré un changement de paradigme. D'appels à projets principalement disciplinaires (qui coexistaient avec des appels thématiques), elle est en effet passée, depuis 2013, à une description en termes de 9 défis de société (1) et d'un défi de tous les savoirs qui s'appuie sur la déclinaison de la Stratégie Nationale de la Recherche (SNR). Si le but affiché de l'ANR reste bien de financer la recherche de base sans ignorer ses capacités d'innovation et d'application, on peut considérer, en faisant un parallèle avec la cristallographie, que la nouvelle grille de lecture par défi est l'espace réciproque de la vision disciplinaire antérieure. À l'issue de cette transformation, les disciplines académiques se trouveront placées à l'interface entre un nombre limité d'objets d'étude donnés comme cibles aux défis. 
La chimie se trouve ainsi principalement représentée dans les défis « Une énergie propre, sûre et efficace ", "Stimuler le renouveau industriel " et "Vie, santé et bien-être ". Cette situation reste néanmoins réductrice, car elle privilégie un nombre limité d'axes d'application reconnus au détriment de l'émergence de nouveaux enjeux. Cette nouvelle déclinaison accompagne la mise en place, sur le plan européen, d'Horizon 2020 qui met en œuvre trois volets complémentaires: Excellence scientifique, Leadership industriel et Défis de société. La notion de recherche ouverte et sans a priori est préservée dans le volet Excellence grâce à l'ERC (2). Mais pour les deux autres volets, l'accent est également mis sur l'objet d'étude, la finalisation de la recherche et son impact socio-économique à court terme.

\section{L'unité de base : le laboratoire, ou l'unité mixte de recherche (UMR)}

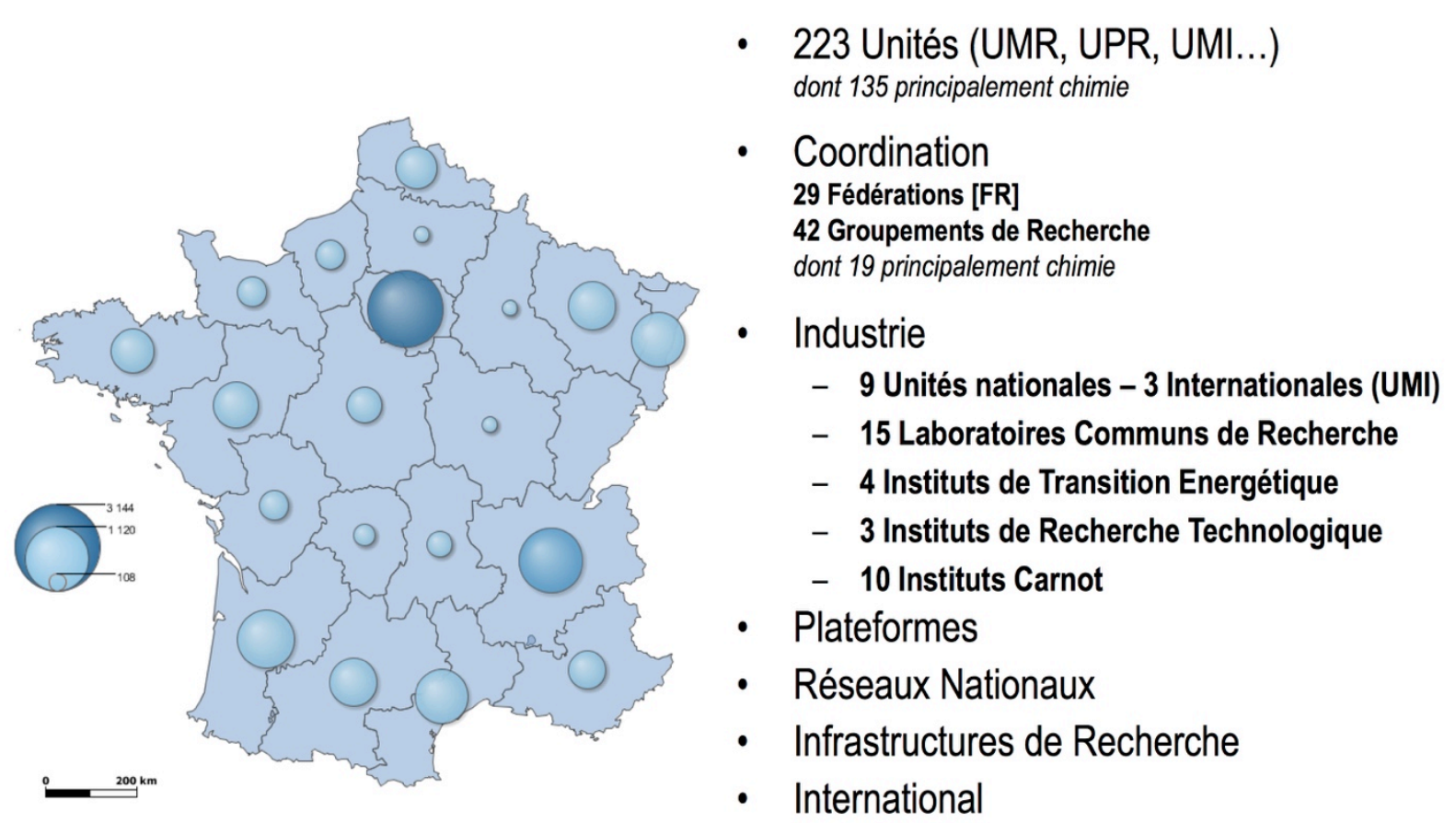

Figure 2 : Répartition nationale des personnels de chimie (universités, écoles, organismes et partenaires) et chiffres-clés des structures de recherche, coordination et réseaux pour la chimie

L'unité de base du fonctionnement de la recherche académique française est le laboratoire ou plutôt l'Unité Mixte de Recherche (UMR), qui rassemble des équipes de recherche (chercheurs, ingénieurs, techniciens) dans un environnement commun et autour d'un projet scientifique partagé identifiant les points forts de l'unité. Ces structures pérennes, mais évolutives, sont créées d'un commun accord par leurs tutelles, qui peuvent être des organismes de recherche (CNRS, Inserm, CEA...), des établissements d'enseignement supérieur (universités, grandes écoles) et/ou des partenaires industriels (grands groupes nationaux et internationaux ou PME). Leurs activités sont suivies par leurs tutelles et évaluées par l'HCERES (3). Sur le plan budgétaire, en ce qui concerne la chimie, on peut considérer aujourd'hui que les tutelles d'une UMR se partagent les frais d'hébergement et les charges salariales et qu'elles assurent ensemble une "dotation de base " qui couvre de 10 à $20 \%$ des coûts de fonctionnement courant de la structure. Dans un paysage désormais 
structuré par les financements sur projet, il est primordial de prendre conscience du fait que ce sont les chercheurs qui lèvent l'essentiel des financements de fonctionnement de leurs équipes dans le cadre des appels à projets publics nationaux (ANR, Région, IdeX, LabeX) et européens (ERC, PCRD, H2020), ainsi que dans le cadre de collaborations avec des industriels.

Les chercheurs en chimie et leurs unités de rattachement sont répartis de façon assez homogène sur l'ensemble du territoire en étroite association avec les établissements d'enseignement supérieur. Ils constituent un maillage territorial fin qui tire sa richesse de sa diversité en se structurant, à l'échelle nationale, autour de nœuds principaux qui ne sont pas nécessairement les sites majeurs de recherche "couronnés " par le PIA. Pour le CNRS et ses partenaires cotutelles des UMR, on comptabilise (en 2014) 220 laboratoires accueillant des chimistes. Cela représente environ 13000 emplois se répartissant de façon équitable entre chercheurs (enseignants-chercheurs et chercheurs: environ 4900 , soit $34 \%$ ), ingénieurs et techniciens (environ 3500 , soit $29 \%$ ) et personnels non permanents (doctorants et post-doctorants) (environ 4 500, soit $37 \%$ ).

Ce sont les enseignants-chercheurs et les chercheurs permanents des laboratoires qui développent leurs thématiques de recherche au sein des unités, dans un contexte interdisciplinaire, même si le découpage disciplinaire prévaut dans la description des activités des organismes ou de celles des établissements. On notera, en particulier, que des interactions ont été établies dans la vie des unités de recherche avec l'ensemble des disciplines qui structurent la recherche académique, y compris avec les sciences humaines. II existe aujourd'hui un continuum interdisciplinaire entre la physique, l'ingénierie, la chimie, les sciences biologiques et les sciences de l'univers et de l'environnement. Cela se traduit par la structuration de communautés scientifiques autour de la chimie-biologie, de la chimiephysique et de la chimie environnementale.

\section{Des outils au service du partage, de la mutualisation et de la collaboration}

Les Fédérations de Recherche (FR), les Groupements de Service (GdS) ou encore les Groupements de Recherche (GdR) sont des outils souples et évolutifs qui structurent les activités des équipes de recherche autour de problématiques disciplinaires ou interdisciplinaires à l'échelle locale comme à l'échelle nationale. On peut prendre pour exemples: les plateformes mutualisées rassemblant des moyens de caractérisation partagés à l'échelle locale ; les réseaux thématiques nationaux colle le Réseau RS2E (4) autour du stockage de l'énergie electrique ; les réseaux d'infrastructures nationales (tels le RMN (5) et le RPE (6)) mettant à disposition de la communauté des instruments au meilleur de leurs performances et dont les coûts sont devenus incompatibles avec un financement par un laboratoire ou un site ; les Très Grandes Infrastructures de Recherche (les TGIR, tels que les synchrotrons ou les lasers à électrons libres)... Les Groupements de Recherche, quant à eux, animent et coordonnent entre elles les diverses communautés scientifiques nationales. Les projets PIA, LabeX et EquipeX ont poussé ces communautés à identifier leurs forces et à se rassembler au niveau local ou au niveau national. 


\section{Les relations entre l'industrie et la recherche académique}

Les relations avec l'industrie sont indissociables de la réalité de la recherche en chimie. Les équipes de recherche conduisent de nombreuses collaborations bilatérales qui reposent avant tout sur des relations personnelles entre chercheurs et ingénieurs appartenant aux deux univers - académique et industriel - sur la base d'un partage réciproque d'expertise et de savoir-faire autour de recherches à la Frontière des Connaissances.

Des relations fortes avec un industriel peuvent s'établir à travers la mise en œuvre de Laboratoires Communs de Recherche (LCR), des structures pérennes, souples et sans mur ou d'Unités Mixtes de Recherche. Ces structures sont construites autour d'une stratégie scientifique décidée en commun. En chimie, on compte 15 LCR (dont certains sont de dimension internationale) et 9 Unités Mixtes (avec de grands groupes ou des PME). De façon remarquable, ces unités développent une stratégie scientifique partagée avec une visibilité académique se situant au plus haut niveau international. Avec Solvay et SaintGobain, ce dispositif a également été porté à l'échelle mondiale, avec trois Unités Mixtes Internationales (UMI), au contact d'Universités ou d'organismes de référence, qui se situent aux États-Unis (COMPASS (7)), en Chine (E2P2 (8)) et au Japon (LINK (9)).

Les laboratoires de recherche académique sont également impliqués dans des dispositifs orientés vers la valorisation socio-économique des résultats de la recherche: 10 Instituts Carnot (10), thématiques, répartis sur l'ensemble du territoire national (Toulouse, Montpellier, Marseille, Grenoble (2 instituts), Lyon, Alsace, Lorraine, Pau, Bordeaux), 4 Instituts pour la Transition Énergétique, les ITE (IPVF (11) à Paris-Saclay, Pivert (12) en Picardie, IFMAS (13) à Lille et IDEEL (14) à Lyon) et 3 Instituts de Recherche Technologique - IRT (Jules Vernes à Nantes, M2P en Lorraine et Saint- Exupéry à Toulouse).

Le CNRS cherche également à évaluer l'impact de la recherche académique en termes de création de start-ups. Dans un communiqué portant sur une étude récente (15), il rapporte la création de plus de 1000 start-ups depuis les années 2000. Pour la chimie, 153 de ces startups sont en activité en 2014 représentant 1000 emplois créés. L'apport sans doute le plus remarquable de cette étude est le fait qu'elle propose une lecture alternative de l'impact de la recherche académique menée dans nos laboratoires. Elle contredit bien des idées reçues et souligne notamment qu'il n'y pas nécessairement d'opposition ou de fossé entre recherche fondamentale et applications industrielles.

Les relations entre les mondes académique et industriel sont très largement réparties dans l'ensemble des laboratoires et l'on doit souligner que le meilleur de ces collaborations est souvent associé au meilleur de la reconnaissance académique (Médailles du CNRS, Académie des Sciences, Collège de France, ERC, articles dans des journaux de premier plan), traduisant ainsi des liens étroits et fructueux qu'exprime mal la représentation linéaire et réductrice des Technology Readiness Levels (TRL).

\section{Les relations internationales}

Les relations internationales sont l'un des principaux moteurs de la reconnaissance des travaux menés par nos laboratoires. Leur participation à la vie de la communauté scientifique internationale est du ressort des chercheurs, qui peuvent participer en tant qu'orateurs ou conférenciers invités à des conférences internationales, mais qui sont également des membres actifs de comités scientifiques ou de comités d'organisation. Les chercheurs sont 
également les relecteurs ou les éditeurs de revues scientifiques publiées par les grands éditeurs et par les grandes sociétés savantes (principalement américaines et anglaises) qui font référence dans notre domaine.

En chimie, comme dans la plupart des autres disciplines, la recherche est internationale et plus de $50 \%$ des publications sont le fruit de collaborations (un peu plus de $25 \%$ avec l'Europe, environ $10 \%$ avec les États-Unis et le Canada, et environ $3 \%$ avec la Chine).

Le paysage du financement des relations internationales reste morcelé, avec des actions du ministère des Affaires étrangères relayées par les ambassades, des appels à projets coordonnés par l'ANR et des initiatives portées par les établissements d'enseignement ou par les organismes de recherche. La mise en place des politiques de site devrait permettre d'en renforcer la cohérence. Le CNRS mène une politique volontariste de soutien aux actions internationales au moyen d'un réseau de bureaux à l'étranger en ce qui concerne les zones d'importance stratégique (Europe, Amérique, Asie) et d'un ensemble de dispositifs coordonnés avec nos partenaires: les Programmes Internationaux de Collaboration Scientifique (PICS), les Laboratoires Internationaux Associés (LIA) et les Groupements de Recherche Internationaux (GdRI) soutiennent des échanges bilatéraux et formalisent les coopérations autour de projets scientifiques. Les Unités Mixtes Internationales (UMI) transposent la notion d'UMR sur le plan international.

Sur le plan européen, la mise en place des programmes Horizon 2020 (H2020) et de leurs déclinaisons en piliers rend indispensable d'être présent non seulement dans les réponses aux appels à projets (en tant que participant ou en tant que coordinateur) en continuant à améliorer la coordination des dispositifs d'aide à la préparation et au suivi des candidatures, mais également d'être actif dans les phases amont de définition des work programs en participant activement aux travaux des Groupes de Travail Nationaux coordonnés par les ministères. Nous avons là une très large capacité de progression.

\section{Les perspectives}

Nous sommes aujourd'hui dans une situation apparemment paradoxale dans laquelle les structures et les financements de la recherche s'inscrivent dans des espaces réciproques, alors qu'in fine, ils poursuivent les mêmes buts et rassemblent les mêmes acteurs. Faut-il regretter un déclin de la vision disciplinaire quand nous déployions tant d'efforts à mettre en œuvre l'interdisciplinarité ? Si l'on veut pousser encore un peu plus loin la métaphore cristallographique, il nous faut nous interroger sur le potentiel que porte cette dualité des points de vue qui considèrent le même objet, la recherche de base, qui est capable de faire émerger de nouveaux concepts en rupture avec les idées établies et les a prioris.

II convient de rappeler que bien des concepts qui nous apparaissent aujourd'hui fondateurs et indiscutables ne sont apparus que tout récemment et qu'ils ont ouvert de nouveaux champs d'application: la chimie supramoléculaire (Nobel 1987), la chimie douce, les matériaux nano-poreux, la réactivité chimique (Nobel 2005), l'électronique organique, la catalyse enzymatique, la chimie théorique et la modélisation (Nobel 2013). Sans être exhaustif, on peut aujourd'hui identifier des domaines particulièrement porteurs dans lesquels la chimie valorise ces avancées: les matériaux carbonés (Flagship Graphene $\mathrm{H} 2020$ ), les matériaux ou les molécules luminescents ou fluorescents, les matériaux actifs ou auto-cicatrisants, le codage et le stockage d'information à l'échelle moléculaire, la conversion 
et le stockage d'énergie, le captage de l'énergie photovoltaïque, le développement de la nano-médecine (imagerie, ciblage, vectorisation), la chimie bio-inspirée et/ou biocompatible, la chimie verte (catalyse écologique et valorisation des déchets), les ressources, la remédiation (dont la phyto-remédiation), les cycles de vie ou encore la chimie de l'atmosphère. La chimie est aux côtés des autres disciplines un acteur majeur de la transition énergétique qui sera au centre de la prochaine Conférence sur le climat de Paris (la COP21). Ces exemples illustrent la capacité créatrice et inventive d'une chimie porteuse de nouveaux concepts et de ruptures qu'il importe de continuer à identifier et à consolider, car ils construisent la chimie de demain.

\section{Notes}

(1) Liste sur le site de l'Agence Nationale de la Recherche.

http://www.agence-nationale-recherche.fr/finances-votre-projet/presentation/

(2) ERC (European Research Council). http://erc.europa.eu

(3) HCERES (Haut Conseil de l'Évaluation de la Recherche et de l'Enseignement Supérieur). http://www.hceres.fr

(4) RS2E (Réseau sur le Stockage Électrochimique de l'Énergie). http://www.energie-rs2e.com/fr

(5) IR-RMN-THC (Infrastructure de Recherche en Résonance Magnétique Nucléaire à Très Hauts Champs). http://www.ir-rmn.fr

(6) RENARD (RÉseau National de RPE interDisciplinaire). http://renard.univ-lille1.fr

(7) COMPASS (Complex Assembly of Soft Matter Laboratory avec Solvay \& University of Pennsylvania). http://www.cnrs-compass.com

(8) E2P2 (Eco-Efficient Products \& Processes Laboratory Shanghaï, en partenariat avec Solvay \& East China Normal University). http://www.e2p2l.com

(9) LINK (Laboratory for Innovative Key Materials and Structures, Tsukuba (Japon) avec Saint Gobain \& NIMS (National Institute for Materials Science)).

(10) Instituts Carnot. http://www.instituts-carnot.eu

(11) Institut PhotoVoltaïque d'Île-de-France. http://www.ipvf.fr

(12) Pivert - Compiègne - Picardie Innovation Végétale Enseignement et Recherches Technologiques. http://www.institut-pivert.com

(13) IFMAS (Lille), Institut Français des Matériaux Agro-Sourcés. http://www.ifmas.eu

(14) IDEEL (Lyon) Chimie matériaux procédés pour l'usine éco-efficiente. http://www.ideel-factory.fr/fr/

(15) Communiqué de presse de 10 décembre 2014. http://www2.cnrs.fr/presse/communique/3837.htm?theme1=10 